\title{
In Vitro Study of Blood Clot Identification and Composition Assessment by Different Magnetic Resonance Sequences
}

Yonghong Ding ${ }^{1}$, Mehdi Abbasi ${ }^{1}$, Yang Liu ${ }^{1}$, Daying Dai ${ }^{1}$, Ramanathan Kadirvel ${ }^{1}$, David F. Kallmes ${ }^{1}$, Waleed Brinjikji ${ }^{2}$

1. Radiology, Mayo Clinic, Rochester, USA 2. Neuroradiology, Mayo Clinic, Rochester, USA

Corresponding author: Waleed Brinjikji, brinjikji.waleed@mayo.edu

\section{Abstract \\ Background}

Growing data suggest that clot composition can impact revascularization outcomes and can potentially guide treatment strategies for stroke patients with large vessel occlusion. We performed an in vitro study to determine which magnetic resonance (MR) signaling characteristics correlate with clot compositions.

\section{Methodology}

A total of 25 clot analogs were prepared by mixing human plasma and red blood cells (RBCs) with five different combinations (five samples for each combination), namely, Group A, fibrin-rich (95\% plasma:5\% RBCs); Group B, fibrin-rich (75\% plasma:25\% RBCs); Group C, intermediate (50\% plasma:50\% RBCs); Group D, RBC-rich (25\% plasma:75\% RBCs), and Group E, RBC-rich (5\% plasma:95\% RBCs). The prepared samples were then scanned with quantitative T2* mapping, T2 fast spin-echo (FSE), T2 gradient-echo (GRE), fluidattenuated inversion recovery (FLAIR), and susceptibility-weighted angiography (SWAN). Thrombus-T2* relaxation time (TT2*RT) and signal intensity (SI) from different scanning sequences were measured in all groups. SIs between different groups were compared using a one-way analysis of variance. Correlation between TT2*RT and SI was determined using the Pearson correlation test.

\section{Results}

The average TT2*RT decreased from $126 \mathrm{~ms}$ to $37 \mathrm{~ms}$ from fibrin-rich to RBC-rich clots (Groups A to E). Mean SIs of Groups D and E were lower than Groups A, B, and C on T2 mapping, T2 FSE, T2 GRE, FLAIR, and SWAN images $(p<0.00001)$. TT2*RT and SI were positively correlated on T2 mapping $(R=0.9628, p=0.009)$.

\section{Conclusion}

Different compositions of blood clots can show different TT2*RT and SI on MR imaging. Quantitative T2* mapping and multicontrast MR scanning can help in the characterization of clots causing large vessel occlusion, which is useful to establish treatment strategies for stroke patients.

Review began 06/22/2021 Review ended 06/29/2021 Published 07/07/2021

\section{(c) Copyright 2021}

Ding et al. This is an open access article distributed under the terms of the Creative Commons Attribution License CC-BY 4.0., which permits unrestricted use, distribution, and reproduction in any medium, provided the original author and source are credited.
Categories: Neurology, Radiology, Neurosurgery

Keywords: stroke, thrombectomy, blood clot, magnetic resonance imaging, susceptibility-weighted imaging

\section{Introduction}

Clot composition can impact revascularization outcomes and strategies in stroke patients with large vessel occlusion. In a preliminary study of 30 patients, a shorter thrombus-T2* relaxation time (TT2*RT) was found to be associated with earlier recanalization after thrombectomy using a combination of stent retriever and aspiration [1]. Other studies have suggested that clots with susceptibility vessel sign (SVS) on T2*-weighted gradient-echo (GRE) imaging are less likely to experience recanalization with intravenous tissuetype plasminogen activator [2]. Because of this potential to use magnetic resonance (MR) imaging for predicting clot composition, we performed an in vitro study to compare different MR imaging sequences for clot composition characterization, which might help to establish and individualize thrombectomy and thrombolytic strategies for patients with acute ischemic stroke.

\section{Materials And Methods}

\section{Preparation of clot analogs}

Following Institutional Review Board approval from Mayo Clinic, whole blood was obtained from volunteers from the Blood Transfusion Service after quality control. Blood was separated into plasma, buffy coat, and erythrocyte-rich layers after centrifugation at 1,500 rounds per minute (RPM) for 12 minutes. The plasma and red blood cells (RBCs) were harvested and recombined in controlled ratios to prepare five different clot 


\section{Cureus}

analogs (five analogs for each combination): Group A, fibrin-rich (95\% plasma:5\% RBCs); Group B, fibrinrich (75\% plasma:25\% RBCs); Group C, intermediate (50\% plasma:50\% RBCs); Group D, RBC-rich (25\% plasma:75\% RBCs); and Group E, RBC-rich (5\% plasma:95\% RBCs). Clotting was initiated by adding 2.06\% calcium chloride $\left(\mathrm{CaCl}_{2}\right)$ solution in a 1:9 ratio $\left(\mathrm{CaCl}_{2}\right.$ solution:blood mixture) followed by thrombin (10 $\mathrm{IU} / \mathrm{mL}$ ) (Sigma-Aldrich, St. Louis, MO, USA) in a 1:1,000 ratio (thrombin:blood mixture). The blood clot mixtures were quickly loaded into $3 \mathrm{~mL}$ plastic Luer-Lock syringes, which were spun at $20 \mathrm{RPM}$ in a hybridization incubator for one hour at $37^{\circ} \mathrm{C}$ to prevent the natural sedimentation of RBCs in static conditions (Figure 1, Panels A-E) [3-5].
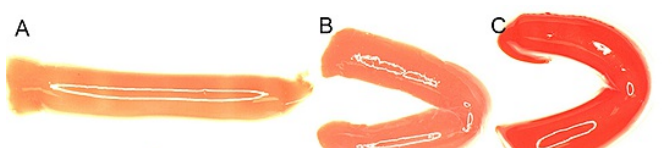

D
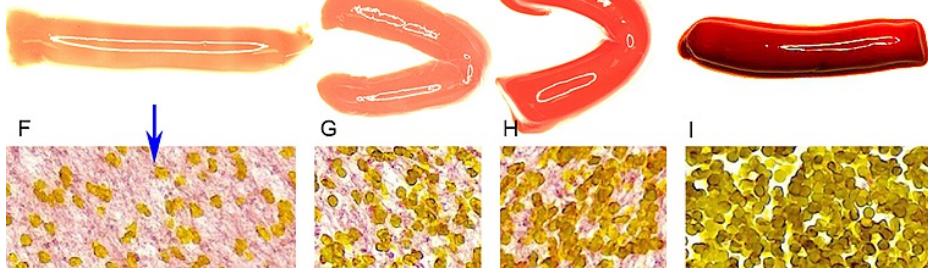

\section{G}
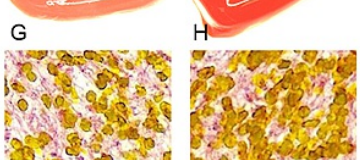

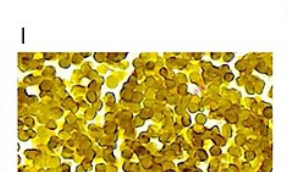

E

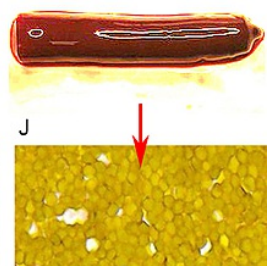

FIGURE 1: Histologic features of clots prepared using different $\mathrm{RBC} /$ fibrin ratios.

A-E: Macrophotographs of clots with different ratios of RBC/fibrin (A, $5 \% \mathrm{RBC}$; $\mathrm{B}, 25 \% \mathrm{RBC}$;, $50 \% \mathrm{RBC}$; $75 \%$ RBC; E, 95\% RBC). The color of clots darkened as the RBC content increased from A to E. F-J: Microphotographs of the clots from A to E, showing more dense distribution of RBCs as the ratio of $\mathrm{RBC} /$ fibrin increased (from scattered RBCs [F, blue arrow] to densely arranged RBCs [J, red arrow]; MSB, original magnification $40 x$ ).

RBC: red blood cell; MSB: Martius Scarlet Blue

\section{Phantom and magnetic resonance scans}

To emulate the attenuation of the brain and skull, the clots were placed in a cylindrical phantom filled with $0.4 \mathrm{mM}$ manganese chloride solution to produce MR image contrast similar to the vessel wall of the human carotid artery. For each clot type, five specimens were placed in the phantom. All 25 clots in the tank were then scanned simultaneously using a 3 Tesla MR imaging scanner (Signa Discovery ${ }^{\mathrm{TM}}$ MR $750 \mathrm{w}, \mathrm{GE}$ Healthcare, Waukesha, WI).

Quantitative T2* mapping and other MR sequences (T2 fast spin-echo [FSE], T2 GRE, fluid-attenuated inversion recovery [FLAIR], and susceptibility-weighted angiography [SWAN]) were used. Specific MR scanning parameters are summarized in Table 1. TT2*RT and MR signal intensities (SIs) were acquired on different images within the selected clot area on a GE HealthCare Advantage Workstation (GE Healthcare, Chicago, IL). 


\section{Cureus}

\begin{tabular}{|c|c|c|c|c|c|c|c|c|c|}
\hline & TR/TE (ms) & $\mathrm{TI}(\mathrm{ms})$ & $\begin{array}{l}\text { Flip angle } \\
\text { (degrees) }\end{array}$ & Echo & $\begin{array}{l}\text { Slice thickness } \\
(\mathrm{mm})\end{array}$ & $\begin{array}{l}\text { Slice gap } \\
(\mathrm{mm})\end{array}$ & $\begin{array}{l}\text { FOV } \\
\text { (cm) }\end{array}$ & Matrix & NEX \\
\hline $\begin{array}{l}\mathrm{T}^{*} \\
\text { mapping }\end{array}$ & $1,200 / 6.8$ & & 90 & $1 / 8$ & 4 & 0.8 & $18 \times 18$ & $\begin{array}{l}320 \times \\
256\end{array}$ & 1.00 \\
\hline T2 FSE & $3,634 / 96.8$ & & 111 & $1 / 1$ & 3 & 0 & $18 \times 18$ & $\begin{array}{l}512 \times \\
512\end{array}$ & 4.00 \\
\hline T2 GRE & 200/8.3 & & 15 & $1 / 1$ & 3 & 0 & $18 \times 18$ & $\begin{array}{l}512 \times \\
512\end{array}$ & 2.00 \\
\hline FLAIR & $11,000 / 144.5$ & $2,633.5$ & 160 & $1 / 1$ & 3.6 & 0 & $22 \times 22$ & $\begin{array}{l}512 \times \\
512\end{array}$ & 1.00 \\
\hline SWAN & $74.6 / 24.2$ & & 15 & $1 / 1$ & 1 & -0.5 & $18 \times 18$ & $\begin{array}{l}384 \times \\
300\end{array}$ & 0.69 \\
\hline
\end{tabular}

TABLE 1: A summary of the parameters of MR sequences.

FOV: field of view; NEX: number of excitations; FSE: fast spin-echo; GRE: gradient-echo; FLAIR: fluid-attenuated inversion recovery; SWAN: susceptibility-weighted angiography

\section{Histologic analysis}

Clot analogs were analyzed histologically to confirm clot composition. Prepared clot analogs were fixed in $10 \%$ phosphate-buffered formalin for two days. Formalin-fixed specimens were embedded in paraffin and cut at a thickness of $3 \mu \mathrm{m}$. Representative slides from each analog were stained with Martius Scarlet Blue (MSB) on which RBC was stained as yellow and fibrin was stained as red (Figure 1, Panels F-J). MSB-stained slides were scanned and analyzed using Aperio ImageScope software (Leica Biosystems, Wetzlar, Germany).

\section{Statistical analysis}

Normality test of TT2*RT and SI was done using the Shapiro-Wilk test. Differences in SI between five groups (Groups A to E) on T2 mapping, T2 FSE, T2 GRE, FLAIR, and SWAN were compared using a one-way analysis of variance (ANOVA). Comparisons of differences in TT2*RT between five groups (Groups A to E) on T2 mapping were performed using the Mann-Whitney U-test. Correlation analysis between TT2*RT and SI values on T2 mapping was performed using the Pearson correlation test. Differences in SI between groups were considered significant if the p-value was less than 0.05 .

\section{Results}

\section{Clot magnetic resonance features on $\mathrm{T}^{*}$ mapping}

The average TT2*RT of the clots with lower RBC content (5\%, 25\%, and 50\%) was $126.2 \pm 21.3,123.4 \pm 19.9$, and $118.8 \pm 20.3 \mathrm{~ms}$, respectively. The value for higher RBC content clots ( $75 \%$ and $95 \%)$ was $42.6 \pm 1.4$ and $37.0 \pm 0.6 \mathrm{~ms}$, respectively (Table 2). As the TT2*RT data did not show normal distribution, the MannWhitney U-test was chosen for further analysis. There were significant differences in TT2*RT between clots with lower and higher RBC content ( $p<0.05$ for all groups). Differences between groups with lower RBC content were not significant $(\mathrm{p}>0.05)$. The TT2*RT from $75 \%$ RBC group was longer than $95 \%$ RBC group ( $p$ $<0.05$ ). Overall, TT2*RT decreased from 126 to $37 \mathrm{~ms}$ from the most fibrin-rich clot to the most RBC-rich clot (about three times higher in the fibrin-rich group) (Figure 2, Panels A-E).

The mean SI value of clots with lower RBC content (5\%, 25\%, and 50\%) on T2 mapping was $1,752 \pm 71,1,723$ \pm 28 , and $1,700 \pm 32$, respectively. The SI value in clots with higher RBC content (75\% and $95 \%$ ) was $1,194 \pm$ 106 and $842 \pm 113$, respectively (Table 2). As the normal distribution of the SI data was confirmed, one-way ANOVA was selected for further comparison. The difference in SI between clots with lower and higher RBC content was significant $(\mathrm{P}<0.00001)$, which indicated the SI from T2 mapping reduced as the RBC content increased (Figure 2, Panels F-J). There was no significant difference in SI values between Groups A, B, and C $(p>0.05)$. The difference in SI between Groups $D$ and $E$ was significant $(p=0.0003)$. There was a strong positive correlation between TT2*RT and SI $(\mathrm{R}=0.9628, \mathrm{p}=0.009)$. 


\section{Cureus}

\begin{tabular}{|c|c|c|}
\hline Groups & $\mathrm{T2}^{*}$ relaxation time $(\mathrm{ms})$ & Signal intensity \\
\hline A & $126.2 \pm 21.3$ & $1,752 \pm 71$ \\
\hline B & $123.4 \pm 19.9$ & $1,723 \pm 28$ \\
\hline C & $118.8 \pm 20.3$ & $1,700 \pm 32$ \\
\hline D & $42.6 \pm 1.4$ & $1,194 \pm 106$ \\
\hline $\mathrm{E}$ & $37.0 \pm 0.6$ & $842 \pm 113$ \\
\hline
\end{tabular}

TABLE 2: T2 mapping relaxation time and signal intensity in the five groups.

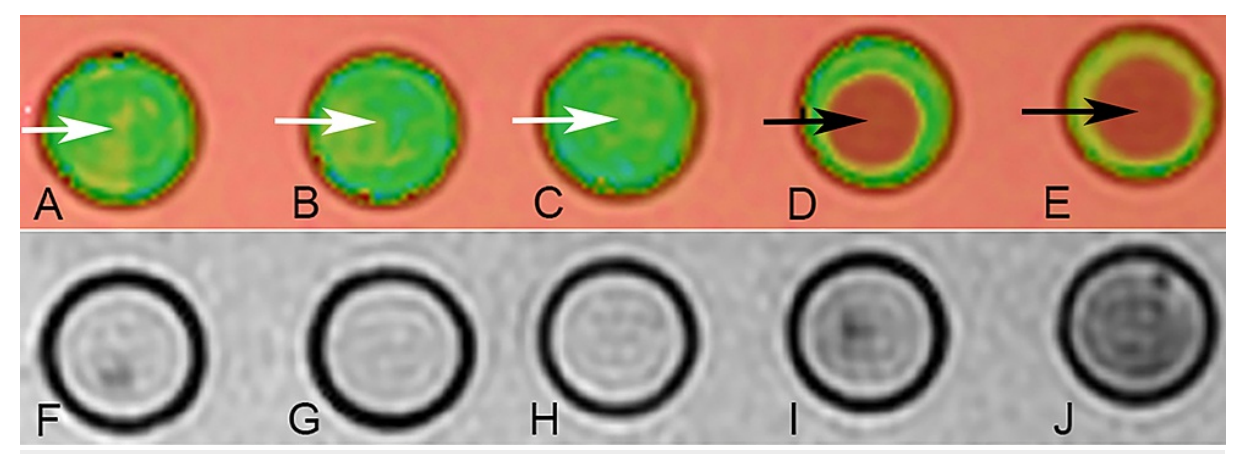

\section{FIGURE 2: MR features from quantitative T2* mapping.}

Relaxation time (A-E) and SI values (F-J). As the RBC content increased, the average $\mathrm{T}^{\star}$ relaxation time decreased from 126 to $37 \mathrm{~ms}$, and the image color changed from green (white arrows) to red (black arrows) in the $\mathrm{T}^{\star}$ estimation images (A to E). SI values dropped as the RBC content increased, and the image became darker ( $F$ to J).

MR: magnetic resonance; SI: signal intensity; RBC: red blood cell

\section{Signal intensity value changes in other magnetic resonance sequences}

Mean SI values of the clots from $5 \%$ to $95 \%$ RBC content were $3,264,3,151,3,075,1,567$, and 928 on T2 FSE imaging; $1,458,1,411,1,392,953$, and 875 on T2 GRE imaging; $1,832,1,785,1,778,858$, and 479 on FLAIR imaging; and 5,756, 5,518, 5,416, 2,586, and 1901 on SWAN imaging; respectively (Table 3). All the SI data fit the normal distribution, hence, one-way ANOVA was used for further comparison. The difference in SI between clots with lower (5-50\%) and higher (75-95\%) RBC content was significant on all comparisons (all p-values less than 0.00001), which indicated that the T2 FSE, T2 GRE, FLAIR, and SWAN SI values decreased as the RBC content increased (Figure 3, Panels A-T). The SI between groups with lower RBC content was not significantly different in all four sequences. The difference in SI between groups with higher RBC content (75\% and 95\%) was not significantly different in all sequences $(\mathrm{p}>0.05)$ except on FLAIR $(\mathrm{p}=0.0003)$.

\begin{tabular}{|c|c|c|c|c|c|}
\hline & 5\% RBC & $25 \%$ RBC & $50 \%$ RBC & 75\% RBC & 95\% RBC \\
\hline T2 FSE & $3,264 \pm 54$ & $3,151 \pm 150$ & $3,075 \pm 104$ & $1,567 \pm 152$ & $928 \pm 120$ \\
\hline T2 GRE & $1,458 \pm 25$ & $1,411 \pm 62$ & $1,392 \pm 19$ & $953 \pm 192$ & $875 \pm 38$ \\
\hline FLAIR & $1,832 \pm 75$ & $1,785 \pm 120$ & $1,778 \pm 75$ & $858 \pm 80$ & $479 \pm 60$ \\
\hline SWAN & $5,756 \pm 163$ & $5,518 \pm 423$ & $5,416 \pm 248$ & $2,586 \pm 744$ & $1,901 \pm 112$ \\
\hline
\end{tabular}

TABLE 3: Signal intensities on other MR sequences.

RBC: red blood cell; FSE: fast spin-echo; GRE: gradient-echo; FLAIR: fluid-attenuated inversion recovery; SWAN: susceptibility-weighted angiography; MR: magnetic resonance 


\section{Cureus}

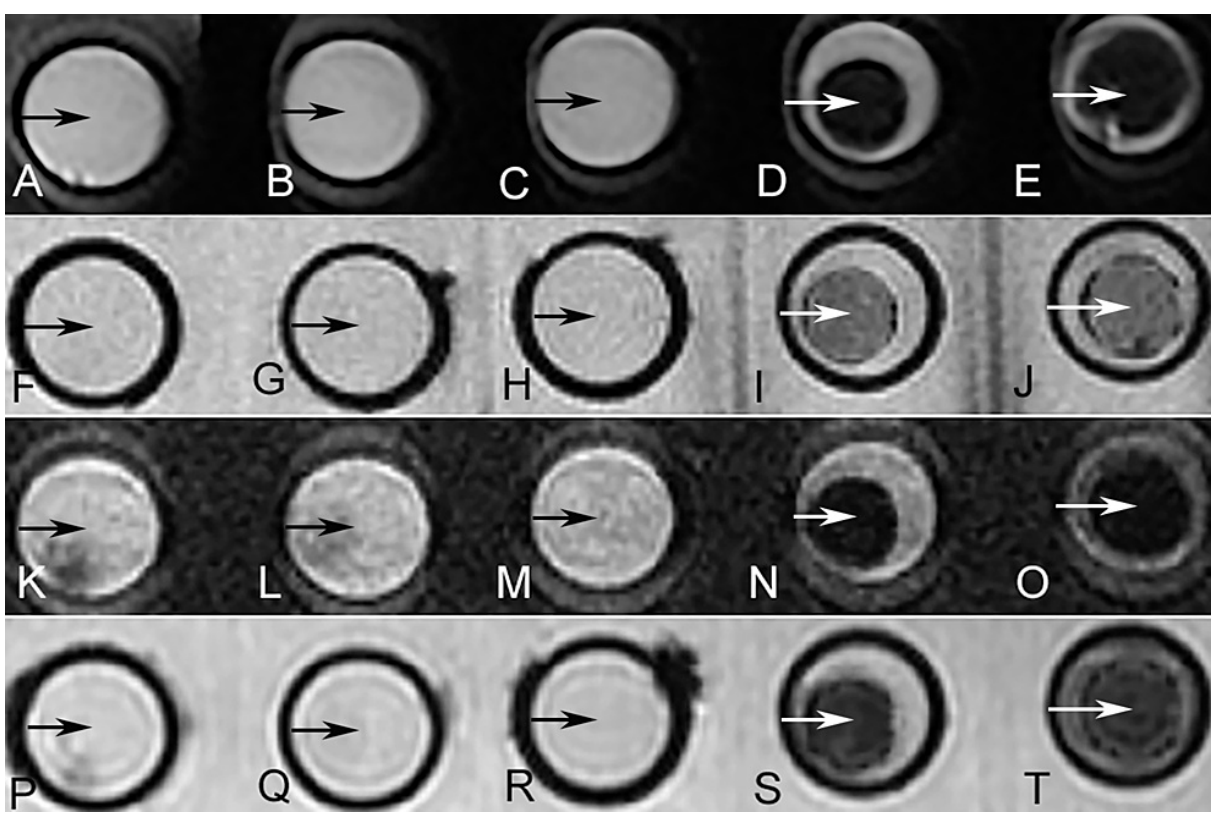

FIGURE 3: MR signals from other scanning sequences.

Images on T2 FSE (from A [5\% RBC] to E [95\% RBC]), T2 GRE (from F [5\% RBC] to J [95\% RBC]), FLAIR (from K [5\% RBC] to O [95\% RBC]), and SWAN (from P [5\% RBC] to T [95\% RBC]). The lower signal is shown in clots with high RBC content (D, E, I, J, N, O, S, T, white arrows) by comparing with low RBC content (A, B, C, F, G, H, K, L, M, P, Q, R, black arrows).

MR: magnetic resonance; FSE: fast spin-echo; RBC: red blood cell; GRE: gradient-echo; FLAIR: fluidattenuated inversion recovery; SWAN: susceptibility-weighted angiography

\section{Discussion}

Our study demonstrated that the TT2*RT of clots decreased as the RBC content increased; hence, T2* mapping can provide more quantitative insight into clot composition. In addition, there was a positive correlation between TT2*RT and SI in the clots with different RBC content. Different MR SIs from different clots with varied RBC content was seen on various MRI sequences (T2 FSE, GRE, FLAIR, and SWAN). Hence, clot characterization can be performed using MR imaging to help guide and individualize both medical and endovascular therapy. An in vitro MR study of clot composition has been reported before [6]. However, our study is more comprehensive as we used multiple scanning sequences for the analysis.

As seen in our study, RBC-rich clots indicated localized hypointense signal at the site of the clot on all T2weighted sequences. This might be caused by hemoglobin from RBCs known as susceptibility effects, which is related to the SVS $[7,8]$. There appears to be a consensus in the literature that the presence of an SVS is associated with improved revascularization outcomes and improved functional outcomes with mechanical thrombectomy $[2,9,10]$. In a study of 180 patients, the presence of the SVS was associated with 2.5 times higher odds of successful revascularization and significant improvements in functional outcomes [10]. In a quantitative study by Bourcier et al., the presence of the SVS was associated with a higher revascularization rate and more favorable clinical outcome with mechanical thrombectomy [11]. Regarding medical therapy, the presence of the SVS was reported to predict lower revascularization rates and poor clinical outcomes in acute stroke patients treated with intravenous tissue plasminogen activator alone [2]. Thus, SVS identification is both reliable and reproducible.

SWAN was reported to be more sensitive than T2*-weighted GRE and T2*-weighted GRE was more sensitive than T2 FSE sequences for the visualization of SVS in the intracranial arteries during the acute phase of ischemic stroke. SWAN is especially helpful to identify small clots [12-16]. In our study, RBC-rich clots were shown as low-signal from all T2-weighted images (irrespective of SWAN), possibly because our clots were not small. If the clot is small, we may need to perform SWAN for clot composition identification. In addition, FLAIR has been reported to show only hyperintense vessel sign in the occluded cerebral vessel [17], which is different from our study because both high (for fibrin-rich clot) and low (for RBC-rich clot) signal could be seen on our FLAIR images. In addition, there was a significant difference in SI between $75 \%$ and $95 \%$ RBCrich clots using FLAIR, which is different from other T2-weighted MR sequences (no significant difference was seen between $75 \%$ and $95 \%$ RBC-rich clots). The underlying mechanisms for this are still unknown.

Hashimoto et al. [18] reported that the interaction between the stent-retriever struts and the clot is likely dependent on the RBC content of the clots. Significantly better patient outcomes can be achieved when the 
entire clot is removed on the first pass without losing any fragments. A fibrin-rich clot can adhere to the vessel wall and is difficult to dislodge using the stent retriever and/or the aspiration catheter; on the other hand, a weakly organized RBC-rich clot can be prone to fragmentation during the stent/catheter pull. Thrombi containing atheromatous gruel might be easily broken into smaller fragments than RBC-rich thrombi and reduce the effectiveness of thrombectomy. Thus an imaging screening protocol to provide an insight to the proportion of RBCs and fibrin in clots would be helpful for selecting a better treatment strategy.

This study has limitations because it was an in vitro study. There was no blood flow around the clots and the clot analogs were not in human cerebral vessels. We acknowledge these limitations of our study, and further investigation should be carried out to address this issue.

\section{Conclusions}

Different TT2*RT and SI values from different compositions of blood clots can be seen on MR imaging. Quantitative T2* mapping and multicontrast MR scanning are useful for the characterization of clots causing large vessel occlusion, which helps to establish treatment strategies for stroke patients.

\section{Additional Information \\ Disclosures}

Human subjects: Consent was obtained or waived by all participants in this study. Mayo Clinic Institutional Review Board issued approval 16-001131. The Mayo Clinic Institutional Board has approved “\#16-001131 Stroke Thromboembolism Registry of Imaging and Pathology.” The reviewer conducted a risk-benefit analysis and determined the study constitutes minimal risk research and this research satisfies the requirement of 45 CFR 46.111. Animal subjects: All authors have confirmed that this study did not involve animal subjects or tissue. Conflicts of interest: In compliance with the ICMJE uniform disclosure form, all authors declare the following: Payment/services info: All authors have declared that no financial support was received from any organization for the submitted work. Financial relationships: All authors have declared that they have no financial relationships at present or within the previous three years with any organizations that might have an interest in the submitted work. Other relationships: All authors have declared that there are no other relationships or activities that could appear to have influenced the submitted work.

\section{Acknowledgements}

Yonghong Ding and Mehdi Abbasi contributed equally to the study. This study was funded by NS105853 (Waleed R01).

\section{References}

1. Bourcier R, Brecheteau N, Costalat V, et al.: MRI quantitative T2* mapping on thrombus to predict recanalization after endovascular treatment for acute anterior ischemic stroke. J Neuroradiol. 2017, 44:241 6. 10.1016/j.neurad.2017.03.006

2. Kimura K, Iguchi Y, Shibazaki K, Watanabe M, Iwanaga T, Aoki J: M1 susceptibility vessel sign on T2* as a strong predictor for no early recanalization after IV-t-PA in acute ischemic stroke. Stroke. 2009, 40:3130-2. 10.1161/STROKEAHA.109.552588

3. Duffy S, Farrell M, McArdle K, et al.: Novel methodology to replicate clot analogs with diverse composition in acute ischemic stroke. J Neurointerv Surg. 2017, 9:486-91. 10.1136/neurintsurg-2016-012308

4. Ding YH, Abbasi M, Michalak G, et al.: Characterization of thrombus composition with multimodality CTbased imaging: an in-vitro study [In Press]. J Neurointerv Surg. 2020, 10.1136/neurintsurg-2020-016799

5. Liu Y, Reddy AS, Cockrum J, et al.: Standardized fabrication method of human-derived emboli with histologic and mechanical quantification for stroke research. J Stroke Cerebrovasc Dis. 2020, 29:105205. 10.1016/j.jstrokecerebrovasdis.2020.105205

6. Bourcier R, Pautre R, Mirza M, et al.: MRI quantitative T2* mapping to predict dominant composition of in vitro thrombus. AJNR Am J Neuroradiol. 2019, 40:59-64. 10.3174/ajnr.A5938

7. Cho KH, Kim JS, Kwon SU, Cho AH, Kang DW: Significance of susceptibility vessel sign on T2*-weighted gradient echo imaging for identification of stroke subtypes. Stroke. 2005, 36:2379-83. 10.1161/01.STR.0000185932.73486.7a

8. Naggara O, Raymond J, Domingo Ayllon M, et al.: T2* "susceptibility vessel sign" demonstrates clot location and length in acute ischemic stroke. PLoS One. 2013, 8:e76727. 10.1371/journal.pone.0076727

9. Bourcier R, Derraz I, Delasalle B, et al.: Susceptibility vessel sign and cardioembolic etiology in the THRACE trial. Clin Neuroradiol. 2019, 29:685-92. 10.1007/s00062-018-0699-8

10. Darcourt J, Withayasuk P, Vukasinovic I, et al.: Predictive value of susceptibility vessel sign for arterial recanalization and clinical improvement in ischemic stroke. Stroke. 2019, 50:512-5. 10.1161/STROKEAHA.118.022912

11. Bourcier R, Mazighi M, Labreuche J, et al.: Susceptibility vessel sign in the ASTER trial: higher recanalization rate and more favourable clinical outcome after first line stent retriever compared to contact aspiration. J Stroke. 2018, 20:268-76. 10.5853/jos.2018.00192

12. Idbaih A, Boukobza M, Crassard I, Porcher R, Bousser MG, Chabriat H: MRI of clot in cerebral venous thrombosis: high diagnostic value of susceptibility-weighted images. Stroke. 2006, 37:991-5. 


\section{Cureus}

10.1161/01.STR.0000206282.85610.ae

13. Bourcier R, Détraz L, Serfaty JM, et al.: MRI interscanner agreement of the association between the susceptibility vessel sign and histologic composition of thrombi. J Neuroimaging. 2017, 27:577-82. 10.1111/jon.12464

14. Allibert R, Billon Grand C, Vuillier F, et al.: Advantages of susceptibility-weighted magnetic resonance sequences in the visualization of intravascular thrombi in acute ischemic stroke. Int J Stroke. 2014, 9:980-4 10.1111/ijs. 12373

15. Leach JL, Strub WM, Gaskill-Shipley MF: Cerebral venous thrombus signal intensity and susceptibility effects on gradient recalled-echo MR imaging. AJNR Am J Neuroradiol. 2007, 28:940-5.

16. Gasparian GG, Sanossian N, Shiroishi MS, Liebeskind DS: Imaging of occlusive thrombi in acute ischemic stroke. Int J Stroke. 2015, 10:298-305. 10.1111/ijs.12435

17. Makkat S, Vandevenne JE, Verswijvel G, et al.: Signs of acute stroke seen on fluid-attenuated inversion recovery MR imaging. AJR Am J Roentgenol. 2002, 179:237-43. 10.2214/ajr.179.1.1790237

18. Hashimoto T, Hayakawa M, Funatsu N, et al.: Histopathologic analysis of retrieved thrombi associated with successful reperfusion after acute stroke thrombectomy. Stroke. 2016, 47:3035-7.

10.1161/STROKEAHA.116.015228 